\title{
High specialization and limited structural change in plant-herbivore networks along a successional chronosequence in tropical montane forest
}

by Redmond, C.M., Auga, J., Gewa, B., Segar, S.T., Miller, S.E., Molem, K., Weiblen, G.D., Butterill, P.T., Maiyah, G., Hood, A.S. and Volf, M.

Copyright, publisher and additional information: Publishers' version. Copyright belongs to the authors.

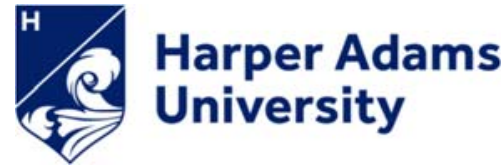




\title{
High specialization and limited structural change in plant- herbivore networks along a successional chronosequence in tropical montane forest
}

\author{
Conor M. Redmond, John Auga, Bradley Gewa, Simon T. Segar, Scott E. Miller, Kenneth Molem, \\ George D. Weiblen, Philip T. Butterill, Gibson Maiyah, Amelia S. C. Hood, Martin Volf, \\ Leonardo R. Jorge, Yves Basset and Vojtech Novotný
}

C. M. Redmond (http://orcid.org/0000-0002-1856-6833) (redmonc1@tcd.ie), S. T. Segar (http://orcid.org/0000-0001-6621-9409), P. T. Butterill (http:// orcid.org/0000-0002-5554-6591), A. S. C. Hood (http://orcid.org/0000-0003-3803-0603), M. Volf (http://orcid.org/0000-0003-4126-3897), Y. Basset (http:/lorcid.org/0000-0002-1942-5717) and V. Novotný (http://orcid.org/0000-0001-7918-8023), Biology Centre, The Czech Academy of Sciences, Inst. of Entomology, Ceske Budejovice, Czech Republic. CMR, STS, PTB, YB and VN also at: Univ. of South Bohemia in Ceske Budejovice, Faculty of Science, Ceske Budejovice, Czech Republic. ASCH also at: Insect Ecology Group, Univ. Museum of Zoology, Cambridge, UK. YB also at: Smithsonian Tropical Research Inst., Panama City, Republic of Panama. MV also at: Molecular Interaction Ecology Group, German Centre for Integrative Biodiversity Research (iDiv) Halle-Jena-Leipzig, Leipzig, Germany. -J. Auga, B. Gewa, K. Molem and G. Maiyah, New Guinea Binatang Research Center, Madang, Papua New Guinea. - S. E. Miller (http://orcid.org/0000-0002-4138-1378), National Museum of Natural History, Smithsonian Inst., Washington, DC, USA. - G. D. Weiblen (http://orcid.org/0000-0002-8720-4887), Bell Museum of Natural History and Dept of Plant Biology, Univ. of Minnesota, Saint Paul, MN, USA. - L. R. Jorge (http://orcid.org/0000-0003-4518-4328), Univ. of Campinas, Dept of Animal Biology, Campinas, Brazil.

\section{Ecography}

42: 162-172, 2019

doi: 10.1111/ecog.03849

Subject Editor: Jose M. Montoya Editor-in-Chief: Miguel Araújo Accepted 5 August 2018
Secondary succession is well-understood, to the point of being predictable for plant communities, but the successional changes in plant-herbivore interactions remains poorly explored. This is particularly true for tropical forests despite the increasing importance of early successional stages in tropical landscapes. Deriving expectations from successional theory, we examine properties of plant-herbivore interaction networks while accounting for host phylogenetic structure along a succession chronosequence in montane rainforest in Papua New Guinea. We present one of the most comprehensive successional investigations of interaction networks, equating to $>40$ person years of field sampling, and one of the few focused on montane tropical forests. We use a series of nine 0.2 ha forest plots across young secondary, mature secondary and primary montane forest, sampled almost completely for woody plants and larval leaf chewers (Lepidoptera) using forest felling. These networks comprised of 12357 plant-herbivore interactions and were analysed using quantitative network metrics, a phylogenetically controlled host-use index and a qualitative network beta diversity measure. Network structural changes were low and specialisation metrics surprisingly similar throughout succession, despite high network beta diversity. Herbivore abundance was greatest in the earliest stages, and hosts here had more species-rich herbivore assemblages, presumably reflecting higher palatability due to lower defensive investment. All herbivore communities were highly specialised, using a phylogenetically narrow set of hosts, while host phylogenetic diversity itself decreased throughout the chronosequence. Relatively high phylogenetic diversity, and thus high diversity of plant defenses, in early succession forest may result in herbivores feeding on fewer hosts than expected. Successional theory, derived primarily from temperate systems, is limited in predicting tropical host-herbivore interactions. All succession stages harbour diverse and unique interaction networks, which together with largely similar network structures and 
consistent host use patterns, suggests general rules of assembly may apply to these systems.

Keywords: ecological gradients, food webs, herbivory.

\section{Introduction}

Examining interaction network properties along ecological gradients is an increasingly popular avenue of research. Such studies provide insights into factors underpinning community assembly and stability (see recent reviews by Tylianakis and Morris 2017, Pellissier et al. 2017). For antagonistic networks, studies commonly focus on spatial change, typically along latitudinal (Novotný et al. 2006, Morris et al. 2014), altitudinal (Morris et al. 2015) or land use gradients (Tylianakis et al. 2007), while temporal change through succession has been comparatively overlooked (but see Villa-Galaviz et al. 2012). This is surprising as ecological succession is not only one of the few community-level processes that we understand and can predict, but secondary regeneration is also increasingly prominent in tropical landscapes, arising through major land use changes and forest disturbance (Chazdon 2014).

Rainforests regenerate by secondary succession in response to natural disturbance events such as treefalls or landslides, and anthropogenic disturbance including selective logging or swidden agriculture. Early regeneration in these gaps is typically dominated by pioneer woody species, possessing distinct life history traits (Turner 2001). Pioneers have shortlived leaves with high nitrogen and water content, photosynthetic capacity and dark respiration rate, while having low mass per area. This contrasts with most mature-forest species which lay on the opposite end of the leaf economics spectrum (Wright et al. 2004). Pioneer plants generally maximize growth rate and, according to the resource availability hypothesis, do so at the expense of protection against herbivores and pathogens, for example by energy-intensive, C-based metabolites (Coley et al. 1985). Fast growing, poorly defended pioneers often suffer higher herbivory and compensate for damage by rapid growth (Fine et al. 2006, Whitfeld et al. 2012b). This well-established ecological theory leads to the assumption that secondary succession is driven by an interplay of plant dispersal and inter-specific competition, with the outcome determined by plant functional traits such as growth rate and dispersal abilities.

While we have a good understanding of successional change, many of the underlying principles have been derived from studies of temperate systems. In temperate zones, early succession communities are often dominated by shortliving herbaceous plant species. This can lead to lower specialisation of herbivores in early succession, where mono- or bivoltine herbivores respond to temporally unpredictable and small-sized pioneer plants, mostly annuals (Novotný 1995). However, Lepš et al. (2001) showed that herbivore specialisation on a subset of host species remained constant during succession in lowland rainforests. In tropical rainforests, even short-lived pioneer trees with a life span $<20$ yr represent a relatively permanent and large resource for their often polyvoltine insect herbivores, obviating a supposed advantage of polyphagy on pioneer vegetation. Thus, how herbivores respond to various succession trajectories, and to what extent general succession theory can be used to predict these responses on the community level, remains unanswered.

We investigate these changes in the context of a montane forest. Such forests generally receive less attention than lowland systems, despite one third of global terrestrial plant diversity being found on mountains (Barthlott et al. 1996). Montane-forest communities are subject to distinct environmental conditions compared with lowland forest, such as lower temperature and land area (Körner 2007). This generates changes in diversity, community composition, functional traits and biotic interactions of plants and herbivores (Sundqvist et al. 2013). Further, studies of forest plantherbivore interactions generally focus on arbitrary subsets of hosts in the community, often phylogenetically controlled (Lepš et al. 2001), limited to common species, and sampled with equal sampling effort (Novotný et al. 2004). These methods arguably generate subjective and somewhat unrealistic representations of real-life networks (Godfray et al. 1999). Thus, we explore successional change using what we refer to as 'whole-forest' networks. A whole-forest approach, i.e. where all woody species in a given area are completely sampled, produces networks which are truly quantitative. Similar proportional biomass sampling approaches are relatively uncommon in host-herbivore interaction network studies, and have focused on quite distinct systems, including temperate forest (Volf et al. 2017), or tropical dry forest communities (Villa-Galaviz et al. 2012).

A whole-forest sampling approach in the tropics will almost invariably encounter a species-rich plant community containing a range of congenerics and distantly related species. As such, understanding host relatedness is important given consumer-resource interactions are largely influenced by evolutionary dynamics of species traits (Futuyma and Agrawal 2009). Herbivores can circumvent only a limited set of plant defensive traits due to genetic, physiological and behavioral constraints (Becerra 2015). Thus, community-wide levels of specialisation may be driven by host community phylogenetic diversity, where diverse defenses drive herbivores towards greater specialisation. Using the recently developed distance based specialisation index (DSI), we may account for the relatedness of hosts in a standardized manner (Jorge et al. 2014, 2017; see methods). More traditional approaches do not account for host phylogeny, but are informative in their own right, and can be seen as complementary. Here, species specificity index (SSI) serves this purpose (Julliard et al. 2006). DSI, however, enables more robust cross-community comparisons between communities with varying levels of community phylogenetic diversity, as is often the case along a successional chronosequence. For example, studies of lowland forest in Papua New Guinea (PNG) have shown that community phylogenetic diversity increases as succession progresses, where early succession communities are typically dominated by a few large genera such as Macaranga and 
Trichospermum (Whitfeld et al. 2012a). The same trend has also been shown in Neotropical forests (Letcher et al. 2012). On the other hand, temperate forests in China followed the opposite trajectory, becoming more phylogenetically uniform with time (Chai et al. 2016).

Here we compile a successional chronosequence of plantherbivore interaction networks through the sampling of distinct phases of succession within a humid tropical montane forest in PNG. By combining established successional theory (Coley et al. 1985 ) with advances in host range and network analyses, we derive several key expectations. 1) Limiting resources in later succession stages promotes investment in host defenses, driving herbivore specialization, increasing DSI and SSI. 2) The greater palatability and nutritional quality of early succession stages will lead to high vulnerability (number of herbivores per host) relative to later stages, and by the same reasoning, to high generality (number of hosts per herbivore). 3) With this finer partitioning of resources, we may also expect late succession networks to have reduced connectance (realised proportion of total potential interactions), while becoming more modular (species organising into strongly interacting subsets delineated by host phylogeny) with time. 4) Finally, higher plant species richness and specialisation in primary forest will result in higher network beta diversity (turnover of network components) than is observed in earlier succession stages due to a more limited pool of pioneer hosts, and a greater prevalence of generalist herbivores.

\section{Materials and methods}

\section{Field site and succession series}

Nine 0.2 ha plots near Yawan village $\left(-6.16388^{\circ} \mathrm{N}\right.$, $\left.146.83833^{\circ} \mathrm{W}\right)$, Morobe Province, Papua New Guinea were sampled using destructive felling at locations earmarked for clearance for swidden subsistence agriculture by the local land-owning community between July 2010 and November 2012. These plots were spatially separated by an average distance of approximately $200 \mathrm{~m}$. Plots were intermingled to avoid pseudoreplication where possible, however potentially hazardous felling conditions and local restrictions limited plot location selection (Supplementary material Appendix 1 Fig. A1). Plots fell within a range of $1720-1860 \mathrm{~m}$ a.s.l. Three distinct phases of succession were identified, namely primary, mature secondary and young secondary forest, based on local accounts regarding previous land use, and plant community structure and composition, where young secondary was $-12-15 \mathrm{yr}$, mature secondary $-25-30 \mathrm{yr}$ and primary forest $>100 \mathrm{yr}$ old. The nine plots comprised of four primary, three mature secondary and two young secondary plots. This approach enabled us to develop a temporal series 'substituting space for time' (Pickett 1989). Before sampling, woody plants with a diameter $>5 \mathrm{~cm}$ dbh were identified to species or morphospecies. Plots were located in a mosaic of primary and secondary forest, where the latter largely results from slash and burn agricultural practices. This subsistence agriculture is small-scale ( $\sim$ ha plots) and low-intensity. Lands are then often abandoned after $2-3 \mathrm{yr}$, allowing natural succession to take place.

\section{Host and herbivore sampling}

Each plot was divided into four $22 \times 22 \mathrm{~m}$ subplots to facilitate sampling in a systematic manner. After clearing the understory, trees $>5 \mathrm{~cm}$ dbh were felled and sampled, beginning with midstory trees. Sampling started from the lowest subplot and proceeded in steps. Trees tangled with lianas had the potential for damaging other trees when felled, thus lianas were cut with machetes where possible. Tree felling was directed into gaps created by previous plot clearance, allowing for easier collection. Collection was carried out immediately upon felling by a team of $\sim 15$ locally recruited collectors supervised by on-site researchers. Collection involved searching for live caterpillars (Lepidoptera), both free feeding and semi-concealed, and placing them in plastic collections pots. In the field lab, trophic links were confirmed with 24-h nochoice feeding trials using host leaves. Specimens were reared to adults where possible and mounted for later taxonomic identification. Identifications were made using existing literature, COI-5P DNA barcoding and dissection of genitalia where necessary. Data are deposited on Genbank (accession numbers KP849894-KP851000), see Miller et al (2015) for further details. Where rearing failed, larvae were preserved in ethanol, morphotyped and a subset (1-11 individuals per morphotype) identified using molecular methods (data are deposited on Genbank accession numbers MK019196MK020093). In total, we attempted to sequence 1187 adults and 1045 larvae. Of these 1132 adults and 897 larvae were successfully barcoded. Limitations on rearing, barcoding, and difficulties in discerning tropical larval Lepidoptera prevented reliable species level identification of the entire community (see Supplementary material Appendix 2 for more details).

Foliage fresh weight of each tree represented a measure of plant resource abundance for herbivores. This was attained by manually stripping trees of their foliage, placing it in sacks and weighing in the field with a hanging scale or electronic balance. Ten leaf discs (diameter $2.3 \mathrm{~cm}$ ) were cut from fresh mature leaves and dried in silica gel for phylogenetic analysis (see below). These discs are stored in $-80^{\circ} \mathrm{C}$ frozen tissue collections at the Univ. of Minnesota (St Paul, Minnesota, USA).

\section{Host phylogenetic diversity}

The host phylogeny was reconstructed using two loci: rbcL, and psbA-trnH, by Bayesian inference (phylogeny is presented and its construction detailed in Supplementary material Appendix 1 Fig. A2). Data deposited on Genbank (accession numbers MH826413-MH826635 and MH826636MH827001). This was used to create a phylogenetic distance matrix from which phylogenetic diversity of host communities, measured as mean pairwise distance (MPD) and mean nearest taxon distance (MNTD), could be determined. MPD is more strongly affected by deep tree topology and 
relationships between distantly related hosts, while MNTD more closely reflects relationships between the tips of the phylogeny (Webb et al. 2002) and thus the presence of alternative, closely related host plant species for herbivores. Both MPD and MNTD were weighted by plant abundance. Standardised effect sizes (SES) were calculated by comparing observed plot means to the plot mean distance under a null distribution. The null model was derived by randomly shuffling species occurrences within the community distance matrix, maintaining total abundance of each species i.e. row sums. This null model is suitable for detecting patterns resulting from species interactions and has a low Type I error rate (Gotelli 2000). Species occurrence differences among sites are assumed to be stochastic. Negative values reflect clustering, while positive values indicate overdispersion. Differences were evaluated using a one-way ANOVA with pairwise contrasts carried out using Tukey post hoc comparison.

\section{Herbivore specialisation}

DSI values were calculated for species within each of the three succession stages following the approach detailed in Jorge et al. $(2014,2017)$. DSI weights the degree of specialisation by the phylogenetic similarity of hosts and their availability, rather than using counts of host species, or higher taxonomic categories. In this sense, DSI measures phylogenetic specialization accounting for differences in the pool of available hosts. The rationale to include the phylogenetic similarity of species within measures of host specialisation is derived from the premise that the more similar a set of species are, the more likely that they will share comparable defensive adaptations. As such, their herbivore communities will encounter familiar costs of use for these resources. In this DSI framework, a specialist is defined as a species that selects a subset of host species more related than is expected by chance. On the other hand, a generalist uses host species that are less related than expected by chance.

The relatedness of host species was measured using MPD, and the deviation from expectations determined using null models that sample the pool of available resources. Here we used a rescaled version of DSI, referred to as DSI*, where differences in abundances and sampling intensities are accounted for, making this measure more amenable to crosscommunity comparisons. The rescaled upper bounds of DSI* were 1 (monophagy) and the lower bounds -1 (maximum generalisation). DSI* was calculated separately for species in young secondary, mature secondary and primary forest. As undersampling can strongly bias estimates of DSI* by inflating the number of monophages, we chose to use only species that were represented by a minimum of ten individuals in each stage. This threshold was chosen as it provides a more accurate reflection of host use, while retaining almost half of the species in the community, and $90 \%$ of individuals. Species specificity index (SSI) was calculated for the same set of herbivores as above (see Julliard et al. 2006, Poisot et al. 2012a for details). This more traditional measure of host use does not account for phylogenetic relatedness. Instead, it quantifies specialization as the coefficient of variation of average herbivore densities among hosts, thus taking into account host diversity and variation in herbivore density among hosts. SSI is bound between 0 and 1, representing low and high specificity respectively. Differences in DSI* and SSI between succession stages were evaluated by linear mixed effect models where species and succession stage were taken as random factors. Significant effects were determined by likelihood ratio test.

\section{Network analysis}

Network structural analyses included all tree species $>5 \mathrm{~cm}$ $\mathrm{dbh}$, and reliably documented herbivore interactions defined as having $\mathrm{n}>1$ observations. Each of the nine plots were characterized by simple species richness measures of lower (host) and higher (herbivore) trophic levels, and quantitative network metrics: 1) Weighted generality, average number of hosts used by each herbivore species, weighted by their marginal totals, 2) Weighted vulnerability, average number of herbivores using each host species, weighted by their marginal totals, 3) Weighted connectance, linkage density (i.e. diversity of interactions per species weighted by marginal totals) divided by the number of species in the network, 4) Modularity, the tendency of interacting species to assemble into strongly interacting subgroups, which interact weakly with species outside of their module (see Supplementary material Appendix 3 for details of these metrics). These metrics cover a range of network properties of interest including structure, stability and specialisation, and were calculated following Bersier et al (2002), Tylianakis et al (2007), Dormann (2009) and Dormann and Strauss (2014). As numerous network metrics are affected by network size (Morris et al. 2014), we accounted for the size of each network within our models by including it as a model covariate. Modularity (Q) is not only affected by network size, but also the number of links and the number of interactions. Thus, to make measures of modularity more amenable to comparison, we compared z-scores standardized by null models obtained by the $\mathrm{r} 2 \mathrm{~d}$ randomization method, which rearranges the interaction matrices keeping marginal sums fixed. These z-scores were then compared across networks (Dormann and Strauss 2014). Differences in network metrics were evaluated using independent models, with habitat type as the explanatory variable (model 1), plus network size as a covariate (model 2). Analyses were performed using the $\mathrm{R}$ statistical environment ver. 3.1.3 (R Development Core Team), with 'bipartite' (Dormann et al 2008) and 'multcomp' (Hothorn et al 2008) packages.

\section{Interaction network beta diversity}

Network beta diversity was partitioned into four components using a modification of the 'betalink' $\mathrm{R}$ package (Poisot et al. 2012b) by Simanonok and Burkle (2014). The complimentary beta diversity measure $(\beta \mathrm{cc})$ was decomposed into 
turnover of plants $(\beta \mathrm{p})$, herbivores $(\beta \mathrm{h})$, both plants and herbivores $(\beta \mathrm{ph})$ and their interactions $(\beta \mathrm{o})$, following Novotný (2009), so that: $\beta c c=\beta p+\beta h+\beta p h+\beta$ o. For more details see Supplementary material Appendix $3 \mathrm{~b}$.

As this is a presence/absence measure of interaction turnover, matrices were converted to binary format for computation. Pairwise contrasts were performed for both within and between succession stages. As we have only two replicate plots in young secondary forest, and thus only a single measure of within stage beta diversity, we omitted this pairwise comparison from the within and between habitat categorical comparisons.

\section{Data deposition}

Host-herbivore interaction data are available Dryad Digital Repository <http://dx.doi.org/10.5061/dryad.bh2rc50> (Redmond et al. 2018). Herbivore barcode sequences can be accessed through BOLD (dx.doi.org/10.5883/DS-YAWAN2) Plant barcode sequences can be accessed through BOLD (dx. doi.org/10.5883/DS-YAWANPL).

\section{Results}

\section{Host and herbivore communities}

830 individual trees from 89 species across 37 families hosted herbivores (Supplementary material Appendix 1 Table A1). Analysis of plant community composition revealed distinct clustering of plots by successional stage (Supplementary material Appendix 1 Fig. A3), with contrasting dominant species (Supplementary material Appendix 1 Fig. A4). Host phylogenetic diversity, measured as MPD, differed between succession stages (ANOVA, $\mathrm{F}_{2,6}=16.15, \mathrm{p}=0.004$ ), where young secondary forest is phylogenetically overdispersed, mature secondary is close to random, becoming significantly less diverse in the phylogenetically clustered primary forest (Fig. 1). When community phylogenetic diversity was measured by MNTD, the same overall trend emerged, however

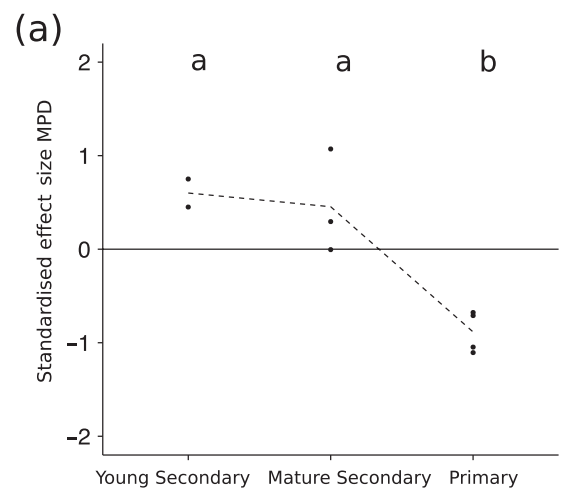

there were no significant effects due to large within stage variance of secondary forest types (ANOVA, $F_{2,6}=1.53$, $\mathrm{p}=0.29$ ).

Mean herbivore abundance was greatest in young secondary forest plots (3046 $0.2 \mathrm{ha}^{-1} \pm 489 \mathrm{SE}$ ), followed by primary forest plots (2461 $0.2 \mathrm{ha}^{-1} \pm 735 \mathrm{SE}$ ) and then mature secondary forest (mean $10870.2 \mathrm{ha}^{-1} \pm 293 \mathrm{SE}$ ). Herbivore abundance per kilogram foliage followed this same order; young secondary $\left(1.29 \mathrm{~kg}^{-1} \pm 0.02 \mathrm{SE}\right)-$ primary $\left(0.97 \mathrm{~kg}^{-1}\right.$ $\pm 0.25 \mathrm{SE}$ ) - mature secondary (mean $0.49 \mathrm{~kg}^{-1} \pm 0.08$ $\mathrm{SE}$. We found no evidence of community-wide seasonality effects, as the numbers of herbivores collected per day per leaf area sampled did not fluctuate notably throughout the collection period (Supplementary material Appendix 1 Fig. A5). Abundance, both total and per unit foliage weight, were not statistically different between succession stages, principally due to an outbreak of two cryptic Leucoma spp. (Erebidae, Lymantriinae) (treated as a single species complex due to difficulties discerning them taxonomically, see Supplementary material Appendix 2 for details). Removal of this Leucoma spp. complex, which represented 4412 individuals found only in primary forest, caused large reductions in mean abundance of primary plots (1359 $\left.0.2 \mathrm{ha}^{-1} \pm 142 \mathrm{SE}\right)$. This leads to differences in total abundance (ANOVA, $\mathrm{F}_{6,2}=17.38, \mathrm{p}=0.003$ ), and abundance $\mathrm{kg}^{-1}$ foliage (ANOVA, $\mathrm{F}_{6,2}=32.7, \mathrm{p}<0.001$ ), where both are significantly higher in young secondary forest than in primary and mature secondary forest.

\section{Network properties and herbivore specialisation}

12357 herbivore individuals, from 292 species across 29 families (Supplementary material Appendix 1 Table A2), were identified to species and used in subsequent network analyses. Representative network plots for each successional stage are presented in Fig. 2. There were significant differences in host richness between succession stages (ANOVA, $\mathrm{F}_{2,6}=11.185, \mathrm{p}=0.009$ ) (Fig. 3a). Host richness was greatest in primary forest plots, however richness did not increase linearly as expected, as young secondary forest was also relatively host rich (Fig. 3a). Herbivore richness also varied along the

(b)

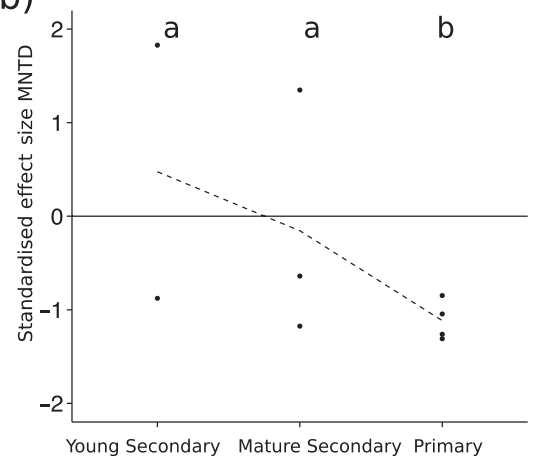

Figure 1. Standardised effect size for weighted mean pairwise distance (MPD) (a) and weighted mean nearest taxon distance (MNTD) (b) of young secondary, mature secondary and primary forest in montane forest in Papua New Guinea. The zero line represents a random structure, while values above zero tend towards overdispersion, and the values below, phylogenetic clustering. Different letters highlight significant differences between succession stages following Tukey post hoc comparisons. Dotted line passes through average values. 


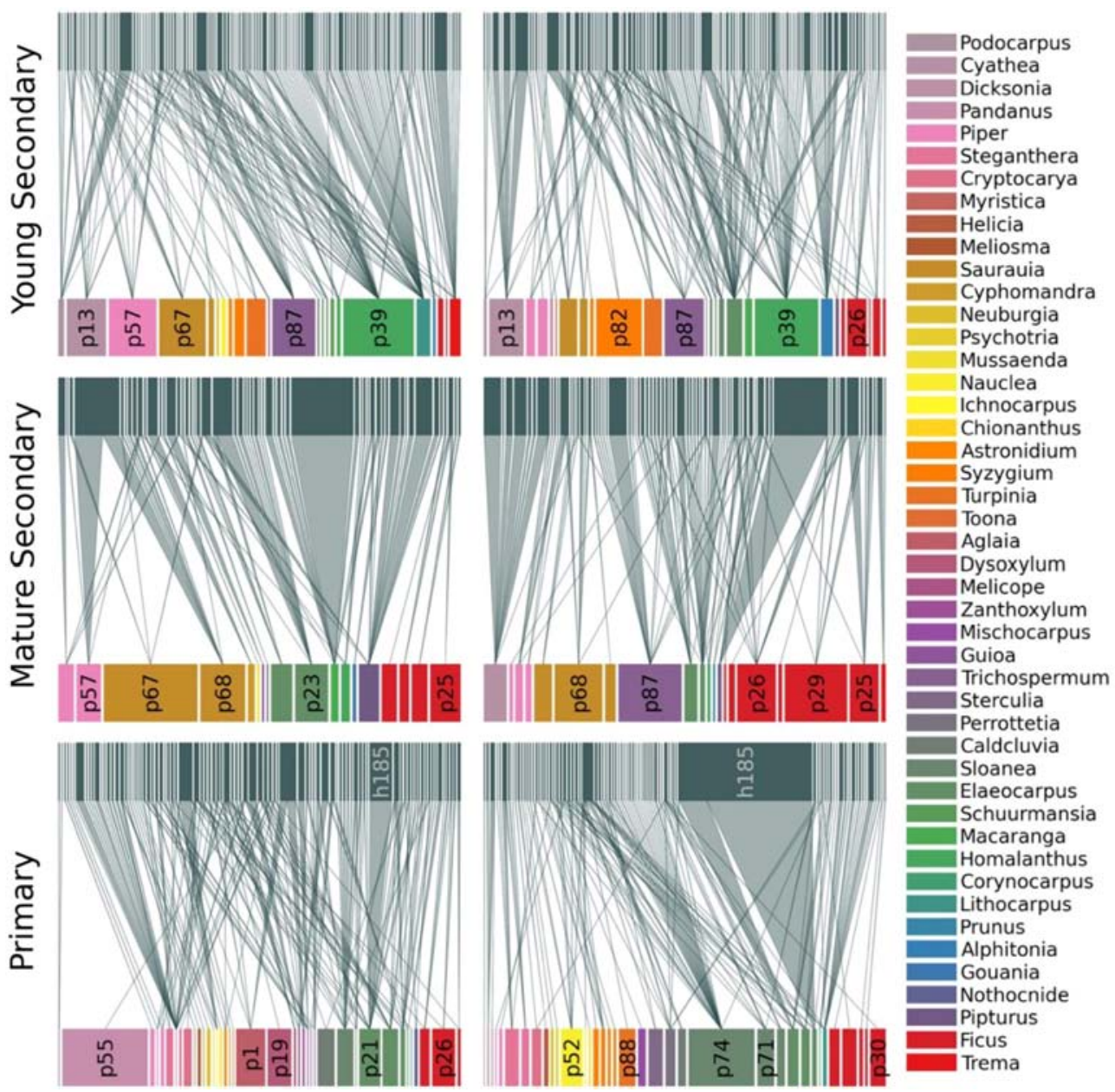

Figure 2. Representative bipartite networks from each of the three stages of succession - young secondary, mature secondary and primary forest. Only two plots per stage are shown for brevity, selected on the basis of network parameters being closest to the mean for that stage. Lower trophic level is coloured according to host genus, and the width of the bars is proportional to host biomass. Hosts are ordered by most basal to most recent (left to right). Only hosts with herbivore interactions are included. The labels of the five most abundant host species in each network is shown, as is the outbreak Leucoma spp. complex (h185+h186). Details of hosts and herbivores are given in Supplementary material Appendix 1 Table A1-A2.

chronosequence (ANOVA, $\mathrm{F}_{2,6}=6.44, \mathrm{p}=0.032$ ), with peak richness in young secondary forest, but again comparable to that of primary forest (Fig. 3b).

Habitat stage had a significant effect on weighted vulnerability and weighted connectance under model 1 (Table 1). These effects diminish after controlling for network size, revealing mature secondary as an intermediate phase, where only pairwise contrasts between young secondary and primary forest remain significant for both vulnerability (Tukey-Kramer, $\mathrm{z}=2.721, \quad \mathrm{p}=0.016$ ) and connectance (Tukey-Kramer, $z=2.786, p=0.013)$. There were no differences in generality when considering successional stage only and when controlling for network size (Table 1, Fig. 3d). Degree distributions of herbivore species can be found in Supplementary material Appendix 1 Fig. A6. All networks were highly modular compared with null models, and succession stage had no effect on modularity (Table 1, Fig. 3f). Herbivore communities were consistently highly phylogenetically specialized (DSI*) (linear mixed effect model, $\chi^{2}=1.46, p=0.481$ ) (Fig. 4a). Three families (Choreutidae, Gelechiidae and Nolidae) were consistently monophagous or near monophagous throughout all stages of succession (Supplementary material Appendix 1 Table A3). Eupterotidae were the least specialized family and were found only in young secondary and mature secondary forest (Table A3). More species-rich families had a broader range of host use, with high specialization being pervasive throughout (Supplementary material Appendix 1 Table A3, Fig. A7). SSI largely reflected DSI*, here however, succession stage had an effect on species specificity (linear mixed effect model, $\left.\chi^{2}=7.27, p=0.026\right)$ where specificity was lower in primary forest (Fig. $4 \mathrm{~b})$.

\section{Beta diversity of networks}

Overall network beta diversity across all pairwise contrasts was high throughout our study system (mean $\beta c c=0.93 \pm$ $0.01 \mathrm{SE}$ ), approaching its upper limits. Overall beta diversity differed significantly between the five pairwise categories, 

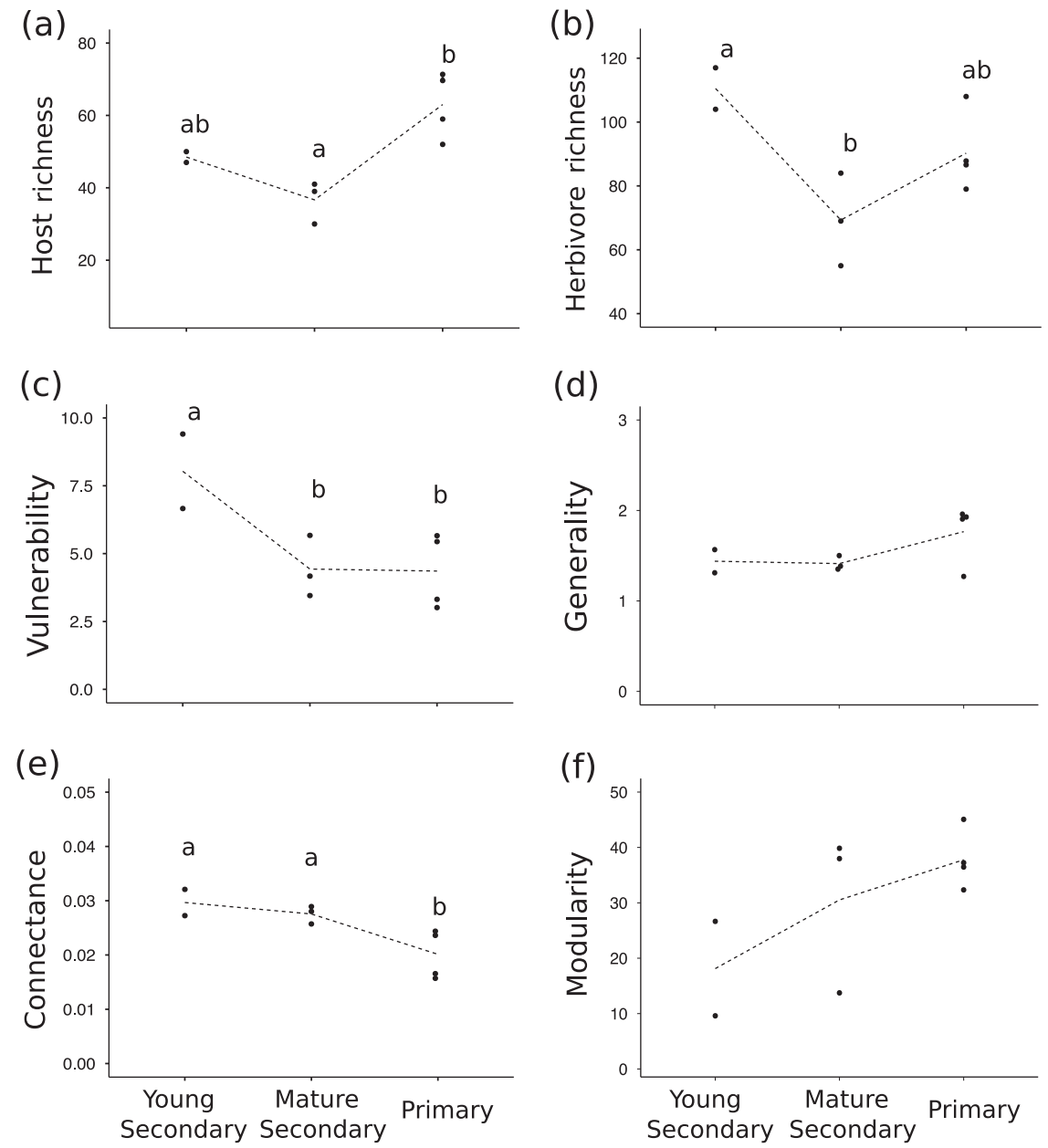

Figure 3. Network metrics for each plot along the successional chronosequence - young secondary, mature secondary and primary forest: (a) host richness, (b) herbivore richness, (c) weighted vulnerability, (d) weighted generality, (e) weighted connectance, and (f) modularity z-scores. Different letters indicate significant differences between succession stages under model 1 . Dotted lines pass through the stage mean.

with pairwise contrasts of different habitat types being significantly higher than within habitat type contrasts (Fig. 5, ANOVA, $\left.\mathrm{F}_{30,4}=12.29, \mathrm{p}<0.001\right)$. Contributions to overall network beta diversity, calculated across all pairwise contrasts, were partitioned into plant species turnover (mean $\beta \mathrm{p}=0.20$ $\pm 0.01 \mathrm{SE}$ ), herbivore turnover (mean $\beta \mathrm{h}=0.23 \pm 0.01 \mathrm{SE}$ ), plant and herbivore turnover (mean $\beta \mathrm{ph}=0.33 \pm 0.02 \mathrm{SE}$ ), and interaction turnover (mean $\beta o=0.16 \pm 0.01 \mathrm{SE}$ ).

\section{Discussion}

Tropical forest succession is a dynamic process where plant species compete for newly available space and resources, resulting in changes to community composition and functional traits (Guariguata and Ostertag 2001, Whitfeld et al. 2014). Despite this, we found that patterns of herbivore host use were more similar than expected and that underlying network properties changed little. Generality did not decrease in primary forest, rather it remained at comparable levels throughout all three stages. This expands the findings of Lepš et al. (2001) to whole communities, where previously only a subset of hosts was examined. Similarly, herbivores in all three succession stages were quite highly specialized when phylogenetic diversity and availability of hosts was taken into account (DSI*). SSI largely reflected this also. However, using SSI, primary forest herbivore specificity was unexpectedly lower than secondary stages, thus overestimating host

Table 1. Effects of succession stage on network metrics when considering succession stage alone (model 1), and controlling for network size (model 2). Asterisks indicate significant differences at $\mathrm{p} \leq 0.05$.

\begin{tabular}{lccccc}
\hline \multirow{2}{*}{ Network Metric } & \multicolumn{2}{c}{ Succession stage } & & \multicolumn{2}{c}{+ Network size } \\
\cline { 2 - 3 } \cline { 5 - 6 } & $\mathrm{F}$ & $\mathrm{p}$ & & $\mathrm{F}$ & $\mathrm{p}$ \\
\hline Weighted vulnerability & 5.128 & $0.050^{*}$ & & 4.879 & 0.067 \\
Weighted generality & 2.106 & 0.202 & & 2.192 & 0.207 \\
Weighted connectance & 5.833 & $0.039^{*}$ & & 4.974 & 0.065 \\
Modularity & 2.365 & 0.175 & & 3.327 & 0.121 \\
\hline
\end{tabular}


(a)

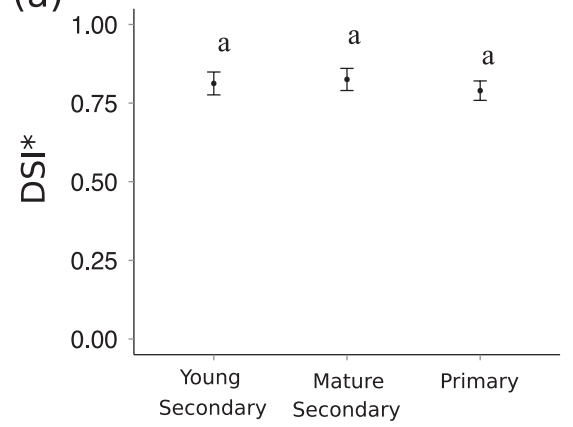

(b)

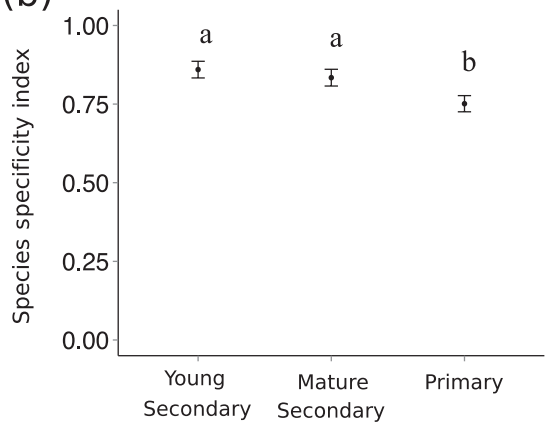

Figure 4. Mean (a) distance based specialisation (DSI*) and (b) species specificity index (SSI) of herbivores along the successional chronosequence - young secondary, mature secondary and primary forest. Error bars represent $+/$ - standard error.

use relative to DSI*. High herbivore specialisation is often reported using both traditional and phylogenetically based measures of specialisation. For instance, lepidopteran communities in Mexican dry forest (Villa-Galaviz et al. 2012) were highly specialized measured using traditional measures, while herbivore communities from four distinct functional groups in PNG were highly phylogenetically specialised (Jorge et al. 2017).

Host plant community composition and the phylogenetic structure of the three succession stages may explain some of these unexpected findings. Phylogenetic diversity decreased with succession, contrasting investigations of lowland systems where succession trajectories typically lead to overdispersion arising from niche differentiation (Whitfeld et al. 2012a). This is likely due to the lack of dominant, diverse genera such as Ficus and Macaranga in the studied secondary montane systems. Given that herbivore communities along the successional chronosequence are similarly phylogenetically limited in their host use, the rather unexpectedly low generality in secondary forest may result from relatively high phylogenetic diversity. Diversity of host defenses closely correlate with their phylogenetic diversity (Agrawal 2011). Despite lower defensive investment in young secondary forest species (Poorter et al 2004, Endara and Coley 2011), a greater diversity of host defenses may prevent herbivores from utilizing multiple hosts in more phylogenetically diverse secondary forest communities. Indeed, host plant chemical diversity was reported to affect herbivory in several cases (Salazar et al. 2016, Massad et al. 2017). Further, phylogenetic limitations to host use have been well documented, typically occurring on the level of host genus, for example; herbivorous communities of lowland forest in PNG (Weiblen et al. 2006), and neotropical dry seasonal forest (Janzen 2003). Thus, in host communities of low phylogenetic diversity, herbivores should have a greater utilizable resource pool, accounting for the decrease in SSI in primary forest herbivores.

Successional theory did however predict some network interactions more accurately. Vulnerability, or the number of herbivore species using a given host, was greatest in young secondary forest. According to the resource availability hypothesis, this finding may reflect greater palatability and nutritional quality of early succession hosts where investment in growth is preferred over defensive investment (Coley et al. 1985, Poorter et al 2004, Endara and Coley 2011). Such hosts have been shown to increase herbivore growth rate and development, shortening maturation to a reproductive state and thus greatly increasing herbivore fitness (Coley et al. 2006). Indeed, we found highest herbivore abundance in young secondary forest, both total, and per unit foliage weight, further indicating a preference for these early succession hosts, and reflecting findings of lowland forest in PNG (Whitfeld et al. 2012b). Network connectance was low throughout all stages of succession. Low connectance is typical of antagonistic networks contrasting that of mutualistic networks, where distinct evolutionary processes generate networks of higher connectance (Thébault and Fontaine 2010). Connectance decreased in primary forest, despite the increased phylogenetic similarity

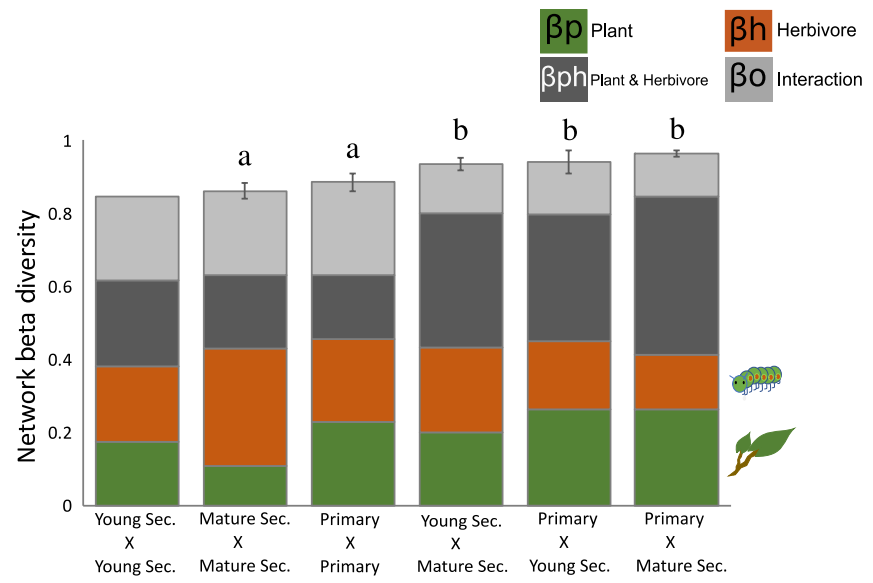

Figure 5. Beta diversity of plant-herbivore networks within and among succession stages. Beta diversity is decomposed into turnover of plants ( $\mathrm{p}=$ green), herbivores $(\mathrm{h}=$ orange), both plants and herbivores ( $\mathrm{ph}=$ dark grey) and their interactions ( $\mathrm{o}=$ light grey). The combined total of all components represents overall network beta diversity. Different letters indicate significant differences between overall beta diversity. Error bars represent 95\% confidence intervals for total network beta diversity for each pairwise comparison. The young secondary $\mathrm{X}$ young secondary pairwise comparison is omitted from statistical analyses due to insufficient replicates. 
of these systems. This is due to greater community-wide resource overlap in secondary forest, where herbivore species are more likely to share the same host. Within primary forest, we see fewer potential niches being realized. If these primary forest hosts are indeed better defended, then we might expect this increased defensive investment would limit the number of herbivores sharing hosts by imposing some ecological costs (Poorter et al 2004, Coley et al. 2006, Zovi et al. 2008). Defensive investment should also generate more modular late succession networks. While all networks across the succession chronosequence were highly modular, quantitative analysis did not reveal a statistical increase in modularity as succession progressed, likely due to low test power. Despite this, there is a clear qualitative trend towards increasing modularity with succession which is ecologically noteworthy. This trend appears to be driven by increases in host species richness rather than changes in herbivore host use, given that phylogenetic specialisation of herbivores throughout succession remains consistently high. The network graphs reveal that many modules have a foundation in a single host species or genus. Segar et al 2017 showed that clades of herbivores tend to interact with clades of hosts in tropical forest. Exploring the changes that occur over time in these groups of strongly interacting species warrants further examination but extends beyond the scope of this study. While antagonistic networks often organise into such modules (Thébault and Fontaine 2010, Cagnolo et al. 2011), this is not always the case (Villa-Galaviz et al. 2012). Understanding modularity, and how module membership changes, is important as a modular structure increases the resilience of networks to the propagation of deleterious domino effects associated with perturbations such as species extinction or local extirpation, and species outbreaks (Stouffer and Bascompte 2011).

While herbivore outbreaks in the tropics can be less conspicuous than in temperate systems, here we encountered an outbreak of a Leucoma spp. complex (Dyer et al 2012). Tropical outbreaks typically arise following prolonged drought, for example, following El Nino events (Van Bael et al. 2004). Indeed, sampling took place in the wake of a moderate $2010 \mathrm{El}$ Nino and during a strong $2011 \mathrm{La}$ Nina event (CPC 2017). Both species within the complex were typically associated with two species of Eleaocarpus hosts. A severe Lepidoptera outbreak occurred in central Panama following the 1997-1998 El Nino, involving at least 12 species (Van Bael et al. 2004). Similarly, these species were also associated with one or two host plant species belonging to the same family. In PNG, coffee plantations have suffered from outbreaks of Tiracola plagiata in the past (Baker 1974). In our system, the outbreak species were exclusively found in primary forest however. Connectance and modularity was lowest and highest respectively in primary forest, and this may have buffered the spread of deleterious effects. Additionally, considering the narrow host-range of this species complex, community-wide effects due to direct competition with other herbivores are likely to be low.
We recognize as a study limitation that plot based network conclusions are drawn from a limited number of replicates and this may introduce biases. However, complete census of interaction networks within 0.2 ha represents a large sampling unit, which is necessary to capture the structure of complex interaction networks in rainforests. The effort needed to obtain these data equates to $>40$ person years in the field. These results provide novel insights regarding large-scale community patterns that may otherwise be overlooked at smaller spatial scales or sampling intensities, where sufficient plant and insect diversity would not be captured. At smaller sampling scales, biases in the form of low within species replication will be introduced, where singleton species dominate the samples. Fayle et al 2015 argue that 'as a guideline, manipulations should mimic the scale at which the focal process or interaction occurs'. While the authors were addressing large-scale experiments, the argument holds equally for large-scale surveys. Nevertheless, it is important to stress that biases due to low sample size may exist. The directionality of these biases for plot-based network metrics is unclear due to novelty of this sampling procedure and lack of comparable studies. However, despite the low number of replicates, clear trends emerge for metrics were variance was low, suggesting patterns are likely robust.

Lepidoptera, as a species-rich herbivore group with a relatively broad host-use spectrum, are a useful and widely-used model taxon. It remains to be seen whether the trends shown here will apply to other herbivore guilds with varying hostuse patterns. However, the extremely high beta diversity both between and within all succession stages would suggest that network structure may be determined by processes which act largely independently of community composition and specific species interactions per se, where perhaps fundamental rules govern assembly of these networks (Morris et al. 2014) or replacement of species occurs between topologically similar species (Dupont et al. 2009). This idea is supported by studies of changes to networks across landscape (Kaartinen and Roslin 2011, Kemp et al. 2017), through time (Kaartinen and Roslin 2012, Kemp et al. 2017) and by comparisons of multiple independent networks across a latitudinal gradient (Morris et al. 2014). Future research directions include developing a perspective of these plant-herbivore interactions which directly accounts for differences in plant traits, and not only host species composition. Traits related to growth and defense, for example specific leaf area and C: $\mathrm{N}$ ratios, can vary both within and between species throughout tropical succession (Poorter et al 2004), with these likely impacting herbivore interactions also.

Promisingly, we show that not just herbivores, but also their interactions and associated ecosystem processes, recover well and rapidly post disturbance, with all stages of succession capable of hosting diverse and unique assemblages. Similarly, studies of a successional chronosequence in tropical dry forest in Mexico reported Lepidoptera herbivore and host networks recovered within six to thirteen years post-disturbance (Villa-Galaviz et al. 2012). Other animal taxa such Coleoptera and nonvolant mammals have been shown to 
recover well within $20-40$ yr post-abandonment, while some, including ants and birds, tend to recover in terms of species richness, while compositional recovery takes longer (Dunn 2004). However, this recovery process will be determined by an interplay of disturbance intensity and landscape characteristics. High-intensity land use, coupled with a lack of seed sources and wildlife refugia, will inevitably slow the recovery process. While a growing list of invertebrates, birds, reptiles and mammals have been shown to recover well, most examples arise from systems well-serviced with influx sources and have experienced relatively low-intensity land use (Dent and Wright 2009). Our system is no different, as slash and burn agriculture is a low-intensity practice, and creates patches of secondary forest in a primary matrix.

Acknowledgements - We thank the community of Yawan for permission to use their land and for their assistance, Binatang Research Centre for field assistance, John Brown, Jeremy Holloway, Jadranka Rota and other taxonomists, Margaret Rosati who organized all the barcoding, and Maria Kuzmina and Univ. of Guelph for sequencing insects and plants.

Funding - This work was supported by the U.S. National Science Foundation grant (DEB 0841885), Grant agency of the Czech Republic (17-23862S), European Research Council (669609) and Jihočeská Univ. Grant Agency (152/2016/P).

Authors contributions - CR led the writing of the manuscript; VN, $\mathrm{YB}, \mathrm{GW}$ and $\mathrm{SM}$ conceived the ideas and designed methodology, JA, BG, PB, KM and GM collected the data; SM, BG, ASCH and CR worked on taxonomy; CR, PB, ASCH, MV, LRJ and STS analysed the data. All authors contributed critically to the drafts and gave final approval for publication.

\section{References}

Agrawal, A. A. 2011. Current trends in the evolutionary ecology of plant defence. - Funct. Ecol. 25: 420-432.

Baker, G.L. 1974. An outbreak of Tiracola Plagiata (Walker) (Lepidoptera:Noctuidae) on coffee in the western highlands district of Papua New Guinea. - Papua New Guinea Agr. 25: 54-58.

Barthlott, W. et al. 1996. Global distribution of species diversity in vascular plants: towards a world map of phytodiversity (Globale Verteilung der Artenvielfalt Höherer Pflanzen: Vorarbeiten zu einer Weltkarte der Phytodiversität). - Erdkunde 50: 317-327.

Becerra, J. X. 2015. On the factors that promote the diversity of herbivorous insects and plants in tropical forests. - Proc. Natl Acad. Sci. USA 112: 6098-6103.

Bersier, L. F. et al 2002. Quantitative descriptors of food-web matrices. - Ecology 83: 2394-2407.

Cagnolo, L. et al. 2011. Network topology: patterns and mechanisms in plant-herbivore and host-parasitoid food webs. - J. Anim. Ecol. 80: 342-351.

Chai, Y. et al. 2016. Patterns of taxonomic, phylogenetic diversity during a long-term succession of forest on the Loess Plateau, China: insights into assembly process. - Sci. Rep. 6: 27087.
Chazdon, R. L. 2014. Second growth: the promise of tropical forest regeneration in an age of deforestation. - Univ. of Chicago Press.

Coley, P. D. et al. 1985. Resource availability and plant antiherbivore defense. - Science 230: 895-899.

Coley, P. D. et al. 2006. The effects of plant quality on caterpillar growth and defense against natural enemies. - Oikos 115: 219-228.

Dent, D. H. and Wright, J. S. 2009. The future of tropical species in secondary forests: a quantitative review. - Biol. Conserv. 142: 2833-2843.

Dormann, C.F. et al. 2008. Introducing the bipartite package: analysing ecological networks. $-\mathrm{R}$ news 8: 8-11.

Dormann, C. F. et al. 2009. Indices, graphs and null models: analyzing bipartite ecological networks. - Open Ecol. J. 2: 7-24.

Dormann, C. F. and Strauss, R. 2014. A method for detecting modules in quantitative bipartite networks. - Methods Ecol. Evol. 5: 90-98.

Dunn, R. R. 2004. Recovery of faunal communities during tropical forest regeneration. - Conserv. Biol. 18: 302-309.

Dupont, Y. L. et al. 2009. Spatio-temporal variation in the structure of pollination networks. - Oikos 118: 1261-1269.

Dyer, L. A. et al. 2012. Insect outbreaks in tropical forests: patterns, mechanisms, and consequences. - In: Barbosa P. et al. (eds), Insect outbreaks revisited. Wiley-Blackwell, pp. 219-245

Endara, M. J. and Coley, P. D. 2011. The resource availability hypothesis revisited: a meta-analysis. - Funct. Ecol. 25: 389-398.

Fayle, T. M. et al 2015. Whole-ecosystem experimental manipulations of tropical forests. - Trends Ecol. Evol. 30: 334-346.

Fine, P. V. A. et al. 2006. The growth - defense trade-off and habitat specialization by plants in Amazonian forests. - Ecology 87: $150-162$.

Futuyma, D. J. and Agrawal, A. A. 2009. Macroevolution and the biological diversity of plants and herbivores. - Proc. Natl Acad. Sci. USA 106: 18054-18061.

Godfray, H. C. et al. 1999. Studying insect diversity in the tropics. - Phil. Trans. R. Soc. B. 354: 1811-1824.

Gotelli, N. J. 2000. Null model analysis of species co-occurrence patterns. - Ecology 81: 2606-2621.

Guariguata, M. R. and Ostertag, R. 2001. Neotropical secondary forest succession: changes in structural and functional characteristics. - For. Ecol. Manage. 148: 185-206.

Hothorn, T, et al 2008. Simultaneous inference in general parametric models. - Biom. J. 50: 346-363.

Janzen, D. H. 2003. How polyphagous are Costa Rican dry forest saturniid caterpillars? - In: Y. Basset et al. (eds), Arthropods of tropical forests: spatio-temporal dynamics and resource use in the canopy. Cambridge Univ. Press.

Jorge, L. R. et al. 2014. An integrated framework to improve the concept of resource specialisation. - Ecol. Lett. 17: $1341-1350$.

Jorge, L. R. et al. 2017. Phylogenetic trophic specialization: a robust comparison of herbivorous guilds. - Oecologia 187: 551-559.

Julliard, R. et al. 2006. Spatial segregation of specialists and generalists in bird communities. - Ecol. Lett. 9: 1237-1244.

Kaartinen, R. and Roslin, T. 2011. Shrinking by numbers: landscape context affects the species composition but not the quantitative structure of local food webs. - J. Anim. Ecol. 80: 622-631. 
Kaartinen, R. and Roslin, T. 2012. High temporal consistency in quantitative food web structure in the face of extreme species turnover. - Oikos 121: 1771-1782.

Kemp, J. E. et al. 2017. Invariant antagonistic network structure despite high spatial and temporal turnover of interactions. - Ecography 40: 1315-1324.

Körner, C. 2007. The use of 'altitude' in ecological research. - Trends Ecol. Evol. 22: 569-574.

Lepš, J. et al. 2001. Habitat and successional status of plants in relation to the communities of their leaf-chewing herbivores in Papua New Guinea. - J. Ecol. 89: 186-199.

Letcher, S. G. et al. 2012. Phylogenetic community structure during succession: evidence from three Neotropical forest sites. - Perspect. Plant Ecol. Evol. Syst. 14: 79-87.

Massad, T. J et al. 2017. Similarity in volatile communities leads to increased herbivory and greater tropical forest diversity. - Ecology 98: 1750-1756.

Miller, S. E. et al. 2015 Barcodes of Lepidoptera Reared from Yawan, Papua New Guinea. - Proc. Ent. Soc. Wash. 117: 247-250

Morris, R. J. et al. 2014. Antagonistic interaction networks are structured independently of latitude and host guild. - Ecol. Lett. 17: 340-349.

Morris, R. J. et al. 2015. Food web structure changes with elevation but not rainforest stratum. - Ecography 38: 792-802.

Novotný, V. 1995. Relationships between life histories of leafhoppers (Auchenorrhyncha-Hemiptera) and their host plants (Juncaceae, Cyperaceae, Poaceae). - Oikos 73: 33-42.

Novotný, V. 2009. Beta diversity of plant-insect food webs in tropical forests: a conceptual framework. - Insect Conserv. Divers. 2: 5-9.

Novotný, V. et al. 2004. No tree an island: the plant - caterpillar food web of a secondary rain forest in New Guinea. - Ecol. Lett. 7: 1090-1100.

Novotný, V. et al. 2006. Why are there so many species of herbivorous insects in tropical rainforests? - Science 313: 1115-1118.

Pellissier, L. et al. 2017. Comparing species interaction networks along environmental gradients. - Biol. Rev. 93: 785-800.

Pickett, S. T. A. 1989. Space-for-time substitution as an alternative to long-term studies. - In: Likens, G. (ed.), Long-term studies in ecology. Springer, pp. 110-135.

Poisot, T. et al. 2012a. A comparative study of ecological specialization estimators. - Methods Ecol. Evol. 3: 537-544.

Poisot, T. et al. 2012b. The dissimilarity of species interaction networks. - Ecol. Lett. 15: 1353-1361.

Poorter, L. et al. 2004. Leaf traits and herbivory rates of tropical tree species differing in successional status. - Plant Biol. 6: 746-754.

Redmond, C. et al. 2018. Data from: High specialization and limited structural change in plant-herbivore networks along a successional chronosequence in tropical montane forest. - Dryad Digital Repository, <http://dx.doi.org/10.5061/dryad.bh2rc50>.

Salazar, D. et al. 2016. Chemical similarity and local community assembly in the species rich tropical genus Piper. - Ecology 97: 3176-3183.

Segar, T. et al. 2017. Variably hungry caterpillars: predictive models and foliar chemistry suggest how to eat a rainforest. - Proc. R. Soc. B 284: 20171803.

Simanonok, M. P. and Burkle, L. A. 2014. Partitioning interaction turnover among alpine pollination networks: spatial, temporal, and environmental patterns. - Ecosphere 5: 1-17.

Stouffer, D. B. and Bascompte, J. 2011. Compartmentalization increases food-web persistence. - Proc. Natl Acad. Sci. USA 108: 3648-3652.

Sundqvist, M. K. et al. 2013. Community and ecosystem responses to elevational gradients: processes, mechanisms, and insights for global change. - Annu. Rev. Ecol. Evol. Syst. 44: 261-280.

Thébault, E. and Fontaine, C. 2010. Stability of ecological communities and the architecture of mutualistic and trophic networks. - Science 329: 853-856.

Turner, I. M. 2001. The ecology of trees in the tropical rain forest. - Cambridge Univ. Press.

Tylianakis, J. M. et al. 2007. Habitat modification alters the structure of tropical host-parasitoid food webs. - Nature 445: 202-205.

Tylianakis, J. M. and Morris, R. J. 2017. Ecological networks across environmental gradients. - Annu. Rev. Ecol. Evol. Syst. 48: 24-48

Van Bael, S. A. et al. 2004. General herbivore outbreak following an El Niño-related drought in a lowland Panamanian forest. - J. Trop. Ecol. 20: 625-633.

Villa-Galaviz, E. et al. 2012. Resilience in plant-herbivore networks during secondary succession. - PLoS One 7: e53009.

Volf, M. et al. 2017. Phylogenetic composition of host plant communities drives plant-herbivore food web structure. - J. Anim. Ecol. 86: 556-565.

Webb, C. O. et al. 2002. Phylogenies and community ecology. - Annu. Rev. Ecol. Evol. Syst. 33: 475-505.

Weiblen, G. D. et al. 2006. Phytogenetic dispersion of host use in a tropical insect herbivore community. - Ecology 87: S62-S75.

Whitfeld, T. J. S. et al. 2012a. Change in community phylogenetic structure during tropical forest succession: evidence from New Guinea. - Ecography 35: 821-830.

Whitfeld, T. J. S. et al. 2012b. Predicting tropical insect herbivore abundance from host plant traits and phylogeny. - Ecology 93: S211-S222.

Whitfeld, T. J. S. et al. 2014. Species richness, forest structure, and functional diversity during succession in the New Guinea lowlands. - Biotropica 46: 538-548.

Wright, I. J. et al. 2004. The worldwide leaf economics spectrum. - Nature 428: 821-827.

Zovi, D. et al. 2008. Ecological costs on local adaptation of an insect herbivore imposed by host plants and enemies. - Ecology 89: 1388-1398.

Supplementary material (Appendix ECOG-03849 at <www. ecography.org/appendix/ecog-03849>). Appendix 1-3. 